\title{
Study on Electro-Magneto-Elastic Fiber Composites with Periodically Distributed Reinforced Phases Under Antiplane Deformation Mode and its Applications
}

\author{
Xingli Ren, Hongli Hou and Yaoling $\mathrm{Xu}^{*}$
}

School of Civil Engineering and Mechanics, Yanshan University, Qinhuangdao 066004, China

\begin{abstract}
The response of electro-magneto-elastic long fiber composites containing periodically distributed reinforced phases under antiplane shear load coupled with inplane electromagnetic load, which lead to the antiplane deformation mode, is dealt with. There are three different electro-magneto-elastic materials in the unit cell (3-phase model), a rigorous analytical method is developed by using the generalized eigenstrains concept integrated with the doubly quasiperiodic Riemann boundary value problem theory. The expressions of electro-magneto-elastic fields in each phase of composites are obtained. As an important application of the presented solution, the effective electro-magneto-elastic moduli are predicted by using the average field theorem and compared with the results derived from the generalized self-consistent method.
\end{abstract}

Keywords: Electro-magneto-elastic composites, 3-phase model, periodically distributed reinforced phases, effective property.

\section{INTRODUCTION}

The electro-magneto-elastic composites, which possess excellent magneto-electric coupling effect, have recently attracted much attention due to the extensive applications for broadband magnetic field probes, electronic packaging, medical ultrasonic imaging, sensors, and actuators [1-2]. Boyd et al. [3] presented a method for using arrays of microelectro-mechanical systems electrodes and electromagnets to achieve micro scale positioning of piezoelectric and piezomagnetic particles in liquid polymers, which would then be solidified to make a polymer matrix magneto-electric composite. This ability would reduce concentrations of stress, electric field, and magnetic field, thereby increasing effective threshold properties such as strength, electric breakdown field, and magnetic saturation field. Recently, by using finite element analysis and volumetric averaging technique, a study on the effective electro-magneto-elastic properties of composites containing a doubly periodic arrangement of piezoelectric and piezomagnetic phases was finished by Lee et al. [4]. In their analysis, complicated periodic boundary conditions of the displacement, electric potential and magnetic potential were subjected to the boundaries of the unit cell. However, the research results have been reported rarely for electro-magneto-elastic composites with periodic microstructures.

The present paper deals with the electro-magneto-elastic composites containing doubly periodic parallelogram arrangement of reinforced phases under antiplane shear load coupled with in plane electromagnetic load, which lead to the antiplane deformation mode. A analytical method is

*Address correspondence to this author at the School of Civil Engineering and Mechanics, Yanshan University, Qinhuangdao 066004, China; Tel: +86-355-8074609; Fax: +86-355-8057101;

E-mails:xylysu@163.com,xylysu@sina.com developed, the effective electro-magneto-elastic moduli are predicted by using the average field theorem and compared with the results derived from the generalized self-consistent method (GSCM).

\section{STATEMENT OF THE PROBLEM}

As shown in Fig. (1a), the parallelogram $\mathrm{P}_{00}$ denotes an unit cell with the boundaries $\Gamma=\Gamma_{1}+\Gamma_{2}+\Gamma_{3}+\Gamma_{4}$, which contains a circular cross-section fiber with an annulus interphase. The unit cell is periodically arranged in the complex plane $z=x_{1}+i x_{2}$, which denote the cross-section of an electro-magneto-elastic composite (see Fig. 1b). In the unit cell, let $\Omega_{f}^{0}, \Omega_{p}^{0}, \Omega_{m}^{0}$ denote the regions occupied by the fiber bounded by the contour $\mathbf{L}_{0}^{0}$, interphase bounded by the contours $L_{0}^{0}, L_{1}^{0}$ and matrix, respectively. $\Omega_{f}, \Omega_{p}, \Omega_{m}$ represent the union of $\Omega_{f}^{0}, \Omega_{p}^{0}, \Omega_{m}^{0}$ and its periodic congruent regions, and $L_{0}, L_{1}$ represent the union of $L_{0}^{0}, L_{1}^{0}$ and its periodic congruent contours, respectively. $R_{0}, R_{1}$ denote the internal and external radius of the interphase annulus. $2 \omega_{1}$ and $2 \omega_{2}$ denote two fundamental periods, the vertices of the unit cell are $\omega_{1}+\omega_{2},-\omega_{1}+\omega_{2},-\omega_{1}-\omega_{2}$ and $\omega_{1}-\omega_{2}$, respectively. The electro-magneto-elastic composite is subjected to the far-field antiplane shear stresses $\tau_{13}^{\infty}, \tau_{23}^{\infty}$ coupled with inplane electrical displacements $D_{1}^{\infty}, D_{2}^{\infty}$ and magnetic inductions $B_{1}^{\infty}, B_{2}^{\infty}$, which lead to the antiplane deformation mode of composites and are not depicted in the Fig. (1b).

\section{ANALYSIS AND SOLUTION}

For an electro-magneto-elastic composite under antiplane shear coupled with in plane electric field and magnetic field, 
(a) a unit cell $\mathrm{P}_{00}$

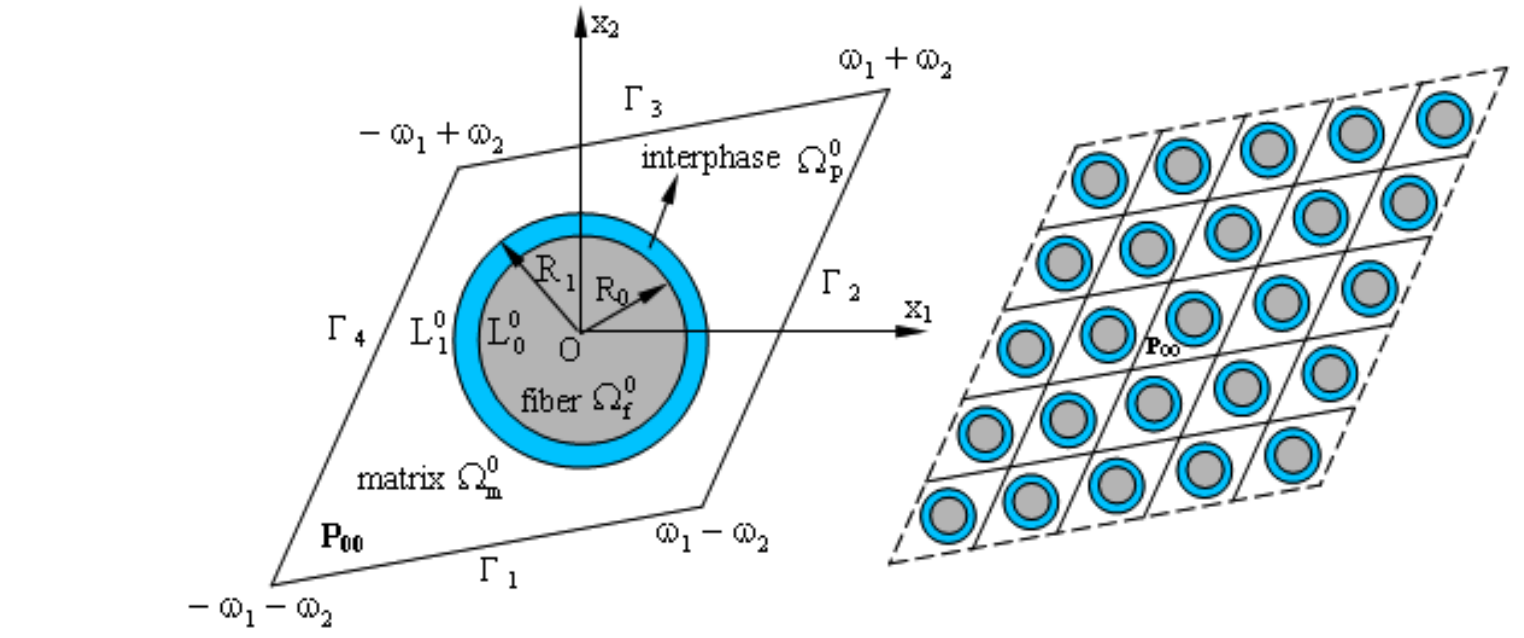

(b) the cross-section of composites

Fig. (1). A unit cell and the cross-section of composites.

there are only the non-trivial antiplane displacement $w$, strain components $\gamma_{13}$ and $\gamma_{23}$, stress components $\tau_{13}$ and $\tau_{23}$, electric potential $\varphi$, electric field components $E_{1}$ and $E_{2}$, electric displacement components $D_{1}$ and $D_{2}$, magnetic potential $\psi$, magnetic field components $H_{1}$ and $H_{2}$, magnetic induction components $B_{1}$ and $B_{2}$, with all field quantities being only the functions of coordinates $x_{1}$ and $x_{2}$. $w, \varphi$ and $\psi$ can be expressed by three analytical potential functions $F(z), \Phi(z)$ and $\Psi(z)$, respectively.

$\mathbf{W}(z)=\operatorname{Re} \mathbf{U}(z)=\frac{1}{2}[\mathbf{U}(z)+\overline{\mathbf{U}(z)}]$

where $\mathbf{W}(z)=\left\{\begin{array}{lll}w(z) & \varphi(z) & \psi(z)\end{array}\right\}^{T}$ is the generalized displacement, $\mathbf{U}(z)=\left\{\begin{array}{lll}F(z) & \Phi(z) & \Psi(z)\end{array}\right\}^{T}$.

The constitutive equations of the problem can be expressed as [5]

$\boldsymbol{\Sigma}_{1}-i \boldsymbol{\Sigma}_{2}=\mathbf{M}\left(\mathbf{Z}_{1}-i \mathbf{Z}_{2}\right)=\mathbf{M} \frac{d \mathbf{U}(z)}{d z}$

where $\Sigma_{k}=\left\{\begin{array}{lll}\tau_{k 3} & D_{k} & B_{k}\end{array}\right\}^{T}$ is the generalized stress, $\mathbf{Z}_{k}=\left\{\begin{array}{lll}\gamma_{k 3} & -E_{k} & -H_{k}\end{array}\right\}^{T}$ is the generalized strain, $k=1,2$.

$\mathbf{M}$ is the property matrix of the electro-magneto-elastic materials

$\mathbf{M}=\left[\begin{array}{ccc}C_{44} & e_{15} & q_{15} \\ e_{15} & -\varepsilon_{11} & -d_{11} \\ q_{15} & -d_{11} & -\mu_{11}\end{array}\right]$

where $C_{44}, \varepsilon_{11}, \mu_{11}$ denote the elastic, dielectric and magnetic permeability moduli, $e_{15}, q_{15}, d_{11}$ denote the piezoelectric, piezomagnetic and magneto electric moduli, respectively.
For the following analysis, we introduce

$\mathbf{T}=-\int_{A}^{B}\left(\boldsymbol{\Sigma}_{2} d x_{1}-\boldsymbol{\Sigma}_{1} d x_{2}\right)=\operatorname{Im}[\mathbf{M U}(z)]_{A}^{B}$

To formulate the problem, the concepts of the generalized eigenstrain and equivalent medium are introduced. Consider two equivalent electro-magneto-elastic cases:

Case 1: An infinite homogeneous electro-magneto-elastic solid with material properties $\mathbf{M}_{m}$ (the same as the matrix) is subjected to uniform far-field generalized stresses $\Sigma_{k}^{\infty}$, from here and afterward, if not mentioned specifically, $k=1,2$. Apparently, in the entire plane, the generalized stresses are uniform, i.e., $\boldsymbol{\Sigma}_{k}^{0}=\boldsymbol{\Sigma}_{k}^{\infty}$. From Eq. (2), the generalized strains $\mathbf{Z}_{k}^{0}=\mathbf{M}_{m}^{-1} \boldsymbol{\Sigma}_{k}^{0}$ are also uniform. For an actual electromagneto-elastic fiber composite, the presence of the doubly periodic regions $\Omega_{f}$ and $\Omega_{p}$ with property matrix $\mathbf{M}_{f}$ and $\mathbf{M}_{p}$ disturbs the uniform generalized strains and stresses. The disturbance field quantities being denoted by $\boldsymbol{\Sigma}_{k, f}^{\prime}, \mathbf{Z}_{k, f}^{\prime}$ in $\Omega_{f}, \quad \boldsymbol{\Sigma}_{k, p}^{\prime}, \mathbf{Z}_{k, p}^{\prime}$ in $\Omega_{p}, \quad \boldsymbol{\Sigma}_{k, m}^{\prime}, \mathbf{Z}_{k, m}^{\prime}$ in $\Omega_{m}$, then total generalized stresses and strains are $\boldsymbol{\Sigma}_{k}^{0}+\boldsymbol{\Sigma}_{k, f}^{\prime}, \mathbf{Z}_{k}^{0}+\mathbf{Z}_{k, f}^{\prime}$ in $\Omega_{f}, \quad \boldsymbol{\Sigma}_{k}^{0}+\boldsymbol{\Sigma}_{k, p}^{\prime}, \mathbf{Z}_{k}^{0}+\mathbf{Z}_{k, p}^{\prime}$ in $\Omega_{p}, \quad \boldsymbol{\Sigma}_{k}^{0}+\boldsymbol{\Sigma}_{k, m}^{\prime}, \mathbf{Z}_{k}^{0}+\mathbf{Z}_{k, m}^{\prime} \quad$ in $\Omega_{m}$, respectively.

Case 2: An infinite homogeneous electro-magneto-elastic solid with property matrix $\mathbf{M}_{m}$, which is the same as the matrix, is subjected to uniform far-field generalized stress $\boldsymbol{\Sigma}_{k}^{\infty}$. Instead of dealing with the presence of actual doubly periodic fibers and interphases with different property matrix $\mathbf{M}_{f}$ and $\mathbf{M}_{p}$, we introduce suitable generalized eigenstrain fields $\mathbf{Z}_{k, f}^{*}$ in $\Omega_{f}$ and $\mathbf{Z}_{k, p}^{*}$ in $\Omega_{p}$, such that the equivalent homogeneous solid has the same electro-magneto-elastic coupling fields. The introduction of the generalized eigenstrains disturbs the uniform electro-magneto-elastic fields, with the disturbance field quantities being denoted by 
$\boldsymbol{\Sigma}_{k, f}^{\prime \prime}, \mathbf{Z}_{k, f}^{\prime \prime}$ in $\Omega_{f}, \boldsymbol{\Sigma}_{k, p}^{\prime \prime}, \mathbf{Z}_{k, p}^{\prime \prime}$ in $\boldsymbol{\Omega}_{p}, \boldsymbol{\Sigma}_{k, m}^{\prime \prime}, \mathbf{Z}_{k, m}^{\prime \prime}$ in $\boldsymbol{\Omega}_{m}$, then total generalized stresses and strains are $\boldsymbol{\Sigma}_{k}^{0}+\boldsymbol{\Sigma}_{k, f}^{\prime \prime}, \mathbf{Z}_{k, f}^{\prime \prime}$ in $\Omega_{f}, \quad \boldsymbol{\Sigma}_{k}^{0}+\boldsymbol{\Sigma}_{k, p}^{n}, \mathbf{Z}_{k}^{0}+\mathbf{Z}_{k, p}^{*}+\mathbf{Z}_{k, p}^{\prime \prime} \quad$ in $\quad \Omega_{p}, \quad \boldsymbol{\Sigma}_{k}^{0}+\boldsymbol{\Sigma}_{k, m}^{\prime \prime}$, $\mathbf{Z}_{k}^{0}+\mathbf{Z}_{k, m}^{\prime \prime}$ in $\Omega_{m}$, respectively.

Let $\mathbf{Z}_{k, f}^{*}+\mathbf{Z}_{k, f}^{\prime \prime}=\mathbf{Z}_{k, f}^{\prime}$ in $\Omega_{f}, \mathbf{Z}_{k, p}^{*}+\mathbf{Z}_{k, p}^{\prime \prime}=\mathbf{Z}_{k, p}^{\prime}$ in $\Omega_{p}$ and $\mathbf{Z}_{k, m}^{\prime \prime}=\mathbf{Z}_{k, m}^{\prime}$ in $\Omega_{m}$, as the generalized eigenstrains $\mathbf{Z}_{k, f}^{*}$ and $\mathbf{Z}_{k, p}^{*}$ are not related to the generalized stresses, the conditions of the equivalence for case 1 and case 2 require

$\left(\mathbf{M}_{f}-\mathbf{M}_{m}\right)\left[\left(\mathbf{Z}_{1}^{0}+\mathbf{Z}_{1, f}^{\prime \prime}\right)-i\left(\mathbf{Z}_{2}^{0}+\mathbf{Z}_{2, f}^{\prime \prime}\right)\right]+\mathbf{M}_{f}\left(\mathbf{Z}_{1, f}^{*}-i \mathbf{Z}_{2, f}^{*}\right)=\mathbf{0}$ in $\Omega_{f}$

$\left(\mathbf{M}_{p}-\mathbf{M}_{m}\right)\left[\left(\mathbf{Z}_{1}^{0}+\mathbf{Z}_{1, p}^{\prime \prime}\right)-i\left(\mathbf{Z}_{2}^{0}+\mathbf{Z}_{2, p}^{\prime \prime}\right)\right]+\mathbf{M}_{p}\left(\mathbf{Z}_{1, p}^{*}-i \mathbf{Z}_{2, p}^{*}\right)=\mathbf{0}$ in $\Omega_{p}$

According to Eqs. (5) and (6), we need to determine four unknown generalized strain vectors $\mathbf{Z}_{1, f}^{\prime \prime}, \mathbf{Z}_{2, f}^{\prime \prime}, \mathbf{Z}_{1, p}^{\prime \prime}, \mathbf{Z}_{2, p}^{\prime \prime}$ induced by the generalized eigenstrain $\mathbf{Z}_{1, f}^{*}, \mathbf{Z}_{2, f}^{*}, \mathbf{Z}_{1, p}^{*}, \mathbf{Z}_{2, p}^{*}$.

$$
\text { Let } \mathbf{W}^{\prime \prime}(z)=\left\{\begin{array}{lll}
w^{\prime \prime}(z) & \varphi^{\prime \prime}(z) & \psi^{\prime \prime}(z)
\end{array}\right\}^{T} \text { and }
$$

$\mathbf{U}^{\prime \prime}(z)=\left\{\begin{array}{lll}F^{\prime \prime}(z) & \Phi^{\prime \prime}(z) & \Psi^{\prime \prime}(z)\end{array}\right\}^{T}$

denote the generalized displacement vector and potential function vector of the electro-magneto-elastic fields induced by the generalized eigenstrain $\mathbf{Z}_{k, f}^{*}$ and $\mathbf{Z}_{k, p}^{*}$, respectively.

Define potential function vector

$\mathbf{U}_{f}^{*}(z)=\left\{\begin{array}{lll}F_{f}^{*}(z) & \Phi_{f}^{*}(z) & \Psi_{f}^{*}(z)\end{array}\right\}^{T}$

corresponding to the generalized eigenstrain $\mathbf{Z}_{k, f}^{*}$ and expand it into Taylor series in $\Omega_{f}^{0}$

$$
\mathbf{U}_{f}^{*}(z)=\left\{\begin{array}{c}
F_{f}^{*}(z) \\
\Phi_{f}^{*}(z) \\
\Psi_{f}^{*}(z)
\end{array}\right\}=\sum_{k=1}^{\infty}\left\{\begin{array}{c}
A_{k}^{f} \\
B_{k}^{f} \\
C_{k}^{f}
\end{array}\right\} z^{k} \quad z \in \Omega_{f}^{0}
$$

From Eq. (2), the generalized eigenstrain can be expressed as

$$
\left(\mathbf{Z}_{1, f}^{*}-i \mathbf{Z}_{2, f}^{*}\right)=\frac{d \mathbf{U}_{f}^{*}(z)}{d z}=\sum_{k=1}^{\infty}\left\{\begin{array}{c}
A_{k}^{f} \\
B_{k}^{f} \\
C_{k}^{f}
\end{array}\right\} k z^{k-1} \quad z \in \Omega_{f}^{0}
$$

Noting Eq. (1), the generalized eigen-displacement vector $\mathbf{W}_{f}^{*}(t)$ can be derived on $L_{0}^{0}$

$$
\begin{aligned}
& \mathbf{W}_{f}^{*}(t)=\left\{\begin{array}{l}
w_{f}^{*}(t) \\
\varphi_{f}^{*}(t) \\
\psi_{f}^{*}(t)
\end{array}\right\}=\frac{1}{2}\left[\mathbf{U}_{f}^{*}(t)+\overline{\mathbf{U}_{f}^{*}(t)}\right] \\
& =\frac{1}{2} \sum_{k=1}^{\infty}\left(\left\{\begin{array}{c}
A_{k}^{f} \\
B_{k}^{f} \\
C_{k}^{f}
\end{array}\right\} t^{k}+\left\{\begin{array}{c}
\overline{A_{k}^{f}} \\
\overline{B_{k}^{f}} \\
C_{k}^{f}
\end{array}\right\} R_{0}^{2 k} \frac{1}{t^{k}}\right)
\end{aligned}
$$

Define potential function vector $\mathbf{U}_{p}^{*}(z)=\left\{\begin{array}{lll}F_{p}^{*}(z) & \Phi_{p}^{*}(z) & \Psi_{p}^{*}(z)\end{array}\right\}^{T}$ corresponding to the generalized eigenstrain $\mathbf{Z}_{k, p}^{*}$, which can be expanded into Laurent series in $\Omega_{p}^{0}$

$$
\mathbf{U}_{p}^{*}(z)=\left\{\begin{array}{c}
F_{p}^{*}(z) \\
\Phi_{p}^{*}(z) \\
\Psi_{p}^{*}(z)
\end{array}\right\}=\sum_{k=1}^{\infty}\left(\left\{\begin{array}{c}
A_{k}^{p} \\
B_{k}^{p} \\
C_{k}^{p}
\end{array}\right\} z^{k}+\left\{\begin{array}{c}
A_{-k}^{p} \\
B_{-k}^{p} \\
C_{-k}^{p}
\end{array}\right\} z^{-k}\right) z \in \Omega_{p}^{0}
$$

The generalized eigenstrain can be expressed as

$$
\left(\mathbf{Z}_{1, p}^{*}-i \mathbf{Z}_{2, p}^{*}\right)=\frac{d \mathbf{U}_{p}^{*}(z)}{d z}=\sum_{k=1}^{\infty} k\left(\left\{\begin{array}{c}
A_{k}^{p} \\
B_{k}^{p} \\
C_{k}^{p}
\end{array}\right\} z^{k-1}-\left\{\begin{array}{c}
A_{-k}^{p} \\
B_{-k}^{p} \\
C_{-k}^{p}
\end{array}\right\} z^{-(k+1)}\right) z \in \Omega_{p}^{0}
$$

The generalized eigen-displacement vector can be derived on $L_{0}^{0}$ and $L_{1}^{0}$

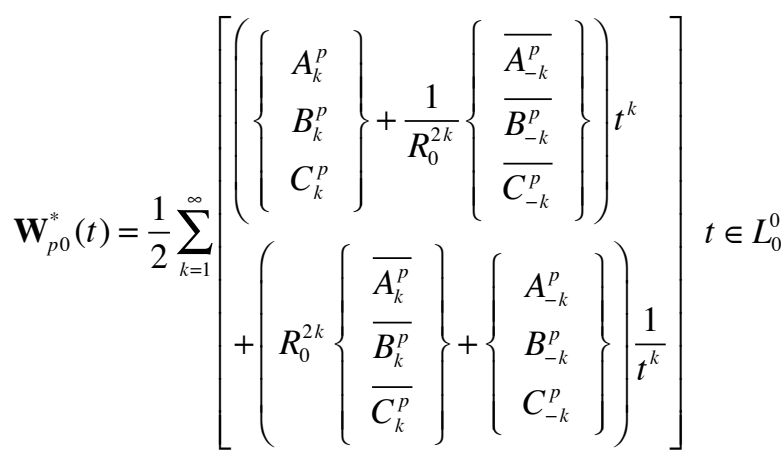

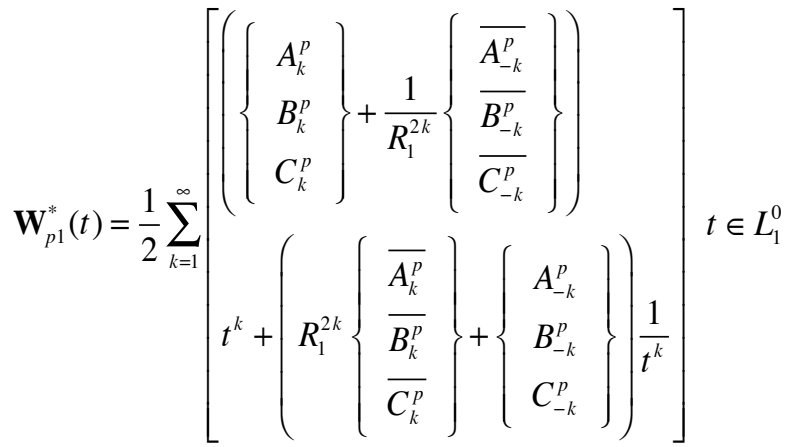


The jump conditions of $\mathbf{W}^{\prime \prime}(z)$ on $L_{0}$ and $L_{1}$ can be written as

$$
\begin{aligned}
& {\left[\mathbf{W}^{\prime \prime}(t)\right]^{+}-\left[\mathbf{W}^{\prime \prime}(t)\right]^{-}=\mathbf{W}_{\mathrm{P} 0}^{*}(t)-\mathbf{W}_{f}^{*}(t) \quad t \in L_{0}} \\
& {\left[\mathbf{W}^{\prime \prime}(t)\right]^{+}-\left[\mathbf{W}^{\prime \prime}(t)\right]^{-}=-\mathbf{W}_{P 1}^{*}(t) \quad t \in L_{1}}
\end{aligned}
$$

where the superscripts "+" and "-" signify the corresponding boundary values of the physical quantities as approached from the interior and the exterior regions of the contour $L_{0}$ or $L_{1}$.

From Eqs. (1) and Eqs. (14), (15), we have

$$
\left(\left[\mathbf{U}^{\prime \prime}(t)\right]^{+}+\overline{\left[\mathbf{U}^{\prime \prime}(t)\right]^{+}}\right)-\left(\left[\mathbf{U}^{\prime \prime}(t)\right]^{-}+\overline{\left[\mathbf{U}^{\prime \prime}(t)\right]^{-}}\right)=2\left[\mathbf{W}_{p 0}^{*}(t)-\mathbf{W}_{f}^{*}(t)\right]
$$$$
t \in L_{0}
$$

$\left(\left[\mathbf{U}^{\prime \prime}(t)\right]^{+}+\overline{\left[\mathbf{U}^{\prime \prime}(t)\right]^{+}}\right)-\left(\left[\mathbf{U}^{\prime \prime}(t)\right]^{-}+\overline{\left[\mathbf{U}^{\prime \prime}(t)\right]^{-}}\right)=-2 \mathbf{W}_{p 1}^{*}(t)$

$t \in L_{1}$

The continuity conditions of the resultant generalized stress on $L_{0}$ and $\mathrm{L}_{1}$ can be written as

$\mathbf{T}^{+}(t)=\mathbf{T}^{-}(t) \quad t \in L_{0}$ and $\mathrm{L}_{1}$

The substitution of Eq. (4) into Eq. (18) yields

$\left(\left[\mathbf{U}^{\prime \prime}(t)\right]^{+}-\overline{\left[\mathbf{U}^{\prime \prime}(t)\right]^{+}}\right)=\left(\left[\mathbf{U}^{\prime \prime}(t)\right]^{-}+\overline{\left[\mathbf{U}^{\prime \prime}(t)\right]^{-}}\right) t \in L_{0}$ and $\mathrm{L}_{1}$

From Eqs. (16), (17) and (19), it is seen that

$$
\begin{aligned}
& {\left[\mathbf{U}^{\prime \prime}(t)\right]^{+}-\left[\mathbf{U}^{\prime \prime}(t)\right]^{-}=\mathbf{W}_{p 0}^{*}(t)-\mathbf{W}_{f}^{*}(t) \quad t \in L_{0}} \\
& {\left[\mathbf{U}^{\prime \prime}(t)\right]^{+}-\left[\mathbf{U}^{\prime \prime}(t)\right]^{-}=-\mathbf{W}_{p 1}^{*}(t) \quad t \in L_{1}}
\end{aligned}
$$

According to the results for the doubly quasi-periodic Riemann boundary problem [6], the general solutions of Eqs. (20) and (21) in unit cell $\mathrm{P}_{00}$ can be expressed as

$$
\mathbf{U}^{\prime \prime}(z)=\left\{\begin{array}{l}
F^{\prime \prime}(z) \\
\Phi^{\prime \prime}(z) \\
\Psi^{\prime \prime}(z)
\end{array}\right\}=\mathbf{C} z+\frac{1}{2 \pi i}\left(\begin{array}{l}
\int_{L_{0}^{0}}\left[\mathbf{W}_{p 0}^{*}(t)-\mathbf{W}_{f}^{*}(t)\right] \zeta(t-z) d t \\
+\int_{L_{1}^{0}}\left[-\mathbf{W}_{P 1}^{*}(t)\right] \zeta(t-z) d t
\end{array}\right)
$$

where $\zeta()$ is Weierstrass Zeta function, $\mathbf{C}=\left\{C_{1}, C_{2}, C_{3}\right\}^{T}$ is complex constant vector to be determined.

Substituting Eqs.(9), (12) and (13) into Eq.(22), U"(z) can be derived

$$
\mathbf{U}^{\prime \prime}(z)= \begin{cases}\mathbf{C} z+\mathbf{U}_{f, 1}(z)+\mathbf{U}_{P, 1}(z)+\mathbf{U}_{P, 3}(z) & z \in \Omega_{\mathrm{f}}^{0} \\ \mathbf{C} z+\mathbf{U}_{f, 2}(z)+\mathbf{U}_{P, 2}(z)+\mathbf{U}_{P, 3}(z) & z \in \Omega_{\mathrm{p}}^{0} \\ \mathbf{C} z+\mathbf{U}_{f, 2}(z)+\mathbf{U}_{P, 2}(z)+\mathbf{U}_{P, 4}(z) & z \in \Omega_{\mathrm{m}}^{0}\end{cases}
$$

where

$$
\begin{aligned}
& \mathbf{U}_{f, 1}(z)=-\frac{1}{2} \sum_{k=1}^{\infty}\left(\left\{\begin{array}{c}
A_{k}^{f} \\
B_{k}^{f} \\
C_{k}^{f}
\end{array}\right\} z^{k}+\left\{\begin{array}{c}
\overline{A_{k}^{f}} \\
\overline{B_{k}^{f}} \\
\overline{C_{k}^{f}}
\end{array}\right\} \frac{(-1)^{k}}{(k-1) !} R_{0}^{2 k}\left[\zeta(z)-\frac{1}{z}\right]^{(k-1)}\right) \\
& \mathbf{U}_{f, 2}(z)=-\frac{1}{2} \sum_{k=1}^{\infty}\left(\left\{\begin{array}{c}
\overline{A_{k}^{f}} \\
\overline{B_{k}^{f}} \\
\overline{C_{k}^{f}}
\end{array}\right\} \frac{(-1)^{k}}{(k-1) !} R_{0}^{2 k} \zeta^{(k-1)}(z)\right)
\end{aligned}
$$

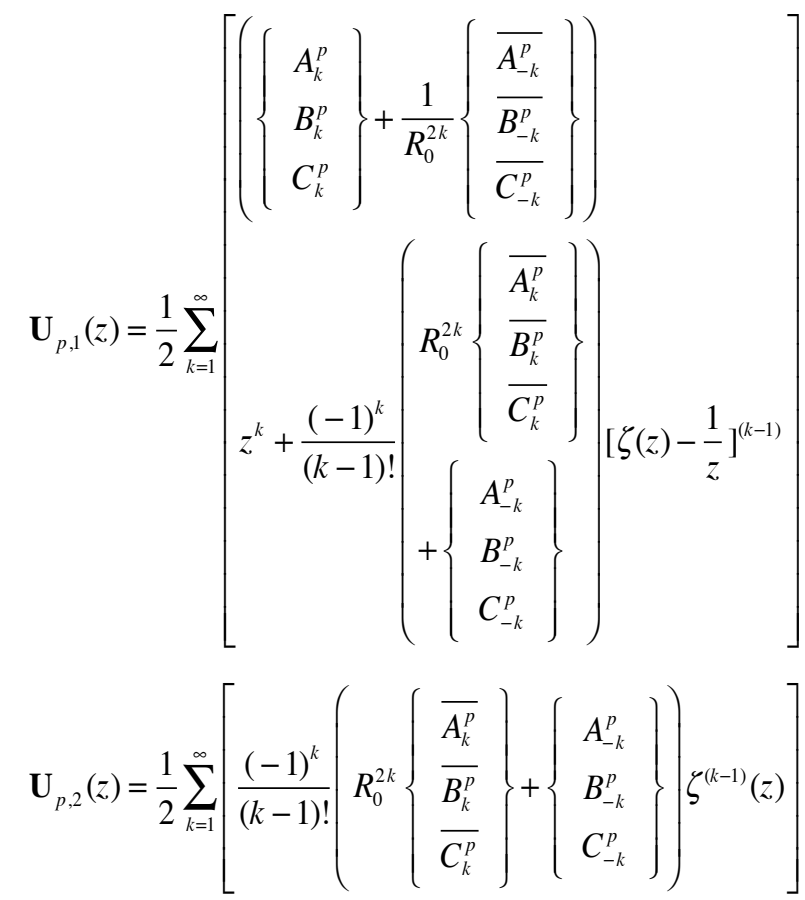

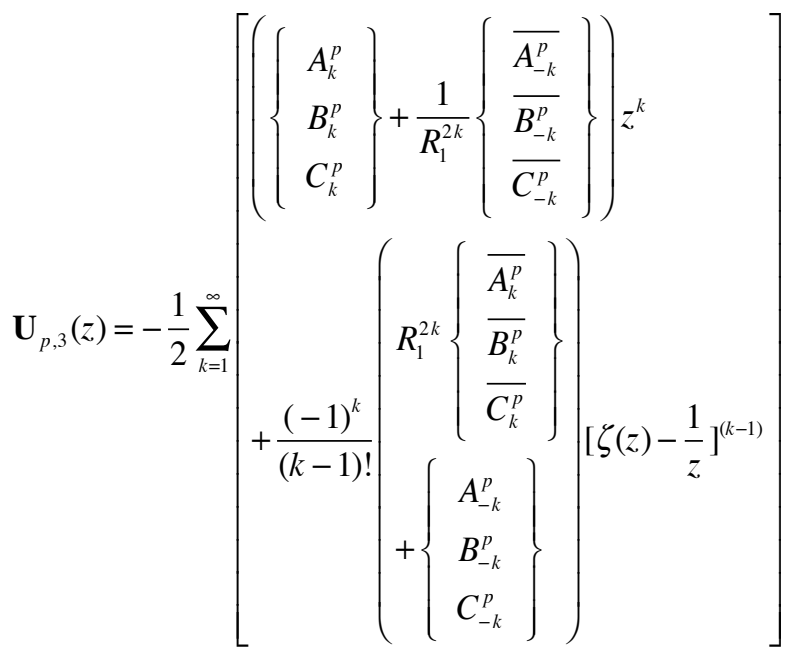


$\mathbf{U}_{p, 4}(z)=-\frac{1}{2} \sum_{k=1}^{\infty}\left[\frac{(-1)^{k}}{(k-1) !}\left(R_{1}^{2 k}\left\{\begin{array}{c}\overline{A_{k}^{p}} \\ \overline{B_{k}^{p}} \\ \overline{C_{k}^{p}}\end{array}\right\}+\left\{\begin{array}{c}A_{-k}^{p} \\ B_{-k}^{p} \\ C_{-k}^{p}\end{array}\right\}\right) \zeta^{(k-1)}(z)\right](29)$

where the superscript $(k-1)$ denotes the $(k-1)$ order derivative with respect to $\mathrm{z}$.

For the generalized eigenstrain problem, no generalized stress at infinity is applied. According to the periodicity, the resultant generalized stress vanishes on each boundary $\Gamma_{k}$ $(k=1,2,3,4)$ of the unit cell $\mathrm{P}_{00}$. From Eq.(4), it follows that

$\left[\mathbf{U}^{\prime \prime}(z)-\overline{\mathbf{U}^{\prime \prime}(z)}\right]_{\Gamma_{k}}=0$

Constant vector $\mathbf{C}$ can be determined by Eq. (30)

$\mathbf{C}=\left\{\begin{array}{l}C_{1} \\ C_{2} \\ C_{3}\end{array}\right\}=\frac{\pi R_{0}^{2}}{2 S}\left(\left\{\begin{array}{c}A_{1}^{f} \\ B_{1}^{f} \\ C_{1}^{f}\end{array}\right\}-\delta_{2}\left\{\begin{array}{l}\overline{A_{1}^{f}} \\ \overline{B_{1}^{f}} \\ \overline{C_{1}^{f}}\end{array}\right\}\right)$

$+\frac{\pi}{2 S}\left(\left(R_{1}^{2}-R_{0}^{2}\right)\left(\left\{\begin{array}{c}A_{1}^{p} \\ B_{1}^{p} \\ C_{1}^{p}\end{array}\right\}-\delta_{2}\left\{\begin{array}{l}\overline{A_{1}^{p}} \\ \overline{B_{1}^{p}} \\ \overline{C_{1}^{p}}\end{array}\right\}\right)\right)$

Noting Eq.(2) and completing the first derivative of $\mathbf{U}^{\prime \prime}(z)$, we can obtain the disturbed generalized strains $\mathbf{Z}_{k, f}^{\prime \prime}$ in $\Omega_{f}^{0}, \mathbf{Z}_{k, p}^{\prime \prime}$ in $\Omega_{p}^{0}, \mathbf{Z}_{k, m}^{\prime \prime}$ in $\Omega_{m}^{0}$, respectively. The complex constants $A_{k}^{f}, B_{k}^{f}, C_{k}^{f}, A_{k}^{p}, B_{k}^{p}, C_{k}^{p}, A_{-k}^{p}, B_{-k}^{p}, C_{-k}^{p}(k=1,2, \ldots)$ can be uniquely determined by Eqs. (5) and (6). Then the total generalized stress and strain in case 1 and case 2 can be obtained, they are equivalent.

\section{ELECTRO-MAGNETO-ELASTIC EFFECTIVE MODULI}

Consider a multiphase electro-magneto-elastic composite subjected to homogeneous generalized stress boundary conditions $\sum^{\infty}$. The average stress theorem of elasticity can be generalized to show

$$
\langle\Sigma\rangle=\sum_{i=1}^{N} \lambda_{i}\left\langle\sum_{i}\right\rangle+\lambda_{m}\left\langle\Sigma_{m}\right\rangle=\Sigma^{\infty}
$$

where the bracket denote the volume average, which can be calculated in the fundamental cell $\mathrm{P}_{00}$ for the doubly periodic problem under consideration. The subscript $m$ denotes the matrix and $i(i=1,2, \ldots, \mathrm{N})$ is used for numbering the dispersed phases. $\lambda_{i}$ denotes $i$-th phase volume fraction.

The effective properties of the electro-magneto-elastic composite are defined as relations between the volume average of the generalized stress and the generalized strain. Noting Eq. (32), we have

$\langle\Sigma\rangle=\Sigma^{\infty}=\mathbf{M}_{*}\langle\mathbf{Z}\rangle$

where $\mathbf{M}_{*}$ is the electro-magneto-elastic effective moduli. $\langle\mathbf{Z}\rangle$ also can be written as the volume-weighted average of $\mathrm{Z}$ over each phase

$$
\begin{aligned}
& \langle\mathbf{Z}\rangle=\sum_{i=1}^{N} \lambda_{i}\left\langle\mathbf{Z}_{i}\right\rangle+\lambda_{m}\left\langle\mathbf{Z}_{m}\right\rangle \\
& \text { Noting } \\
& \left\langle\mathbf{Z}_{m}\right\rangle=\mathbf{M}_{m}^{-1}\left\langle\sum_{m}\right\rangle,\left\langle\mathbf{Z}_{i}\right\rangle=\mathbf{M}_{i}^{-1}\left\langle\sum_{i}\right\rangle
\end{aligned}
$$

from Eqs. (32)-(34), it is obtained that

$$
\left(\mathbf{M}_{*}^{-1}-\mathbf{M}_{m}^{-1}\right) \sum^{\infty}=\sum_{i=1}^{N} \lambda_{i}\left(\mathbf{M}_{i}^{-1}-\mathbf{M}_{m}^{-1}\right)\left\langle\sum_{i}\right\rangle
$$

\section{EXAMPLES AND ANALYSIS}

Electro-magneto-elastic effective moduli play an important role in the design of electro-magneto-elastic composites. Once the averaged generalized stress is determined in each dispersed phase by the present method, electro-magneto-elastic effective moduli $M_{*}$ can be determined by Eq. (36). As an important application of the presented solution, the effective electro-magneto-elastic moduli are predicted and compared with the results derived from the GSCM. Material properties are listed in Table $\mathbf{1 .}$

Example 1: Consider two arrays of reinforced phases, i.e., the square and hexagonal arrays as shown in Fig. (2).

Table 1. Material Properties

\begin{tabular}{|c|c|c|c|}
\hline & BaTiO $_{3}$ (Piezoelectric) & $\mathbf{C o F e}_{2} \mathbf{O}_{4}$ (Piezomagnetic) & Epoxy (Pure Elastic) \\
\hline \hline$C_{44}(\mathrm{GPa})$ & 43 & 45.3 & 0 \\
\hline$e_{15}\left(C / m^{2}\right)$ & 11.6 & 0 & 0 \\
\hline$q_{15}(N / \mathrm{Am})$ & 0 & 550.0 & 0.1 \\
\hline$\varepsilon_{11}\left(10^{-9} C^{2} / N m^{2}\right)$ & 11.2 & 0.08 & 0 \\
\hline$d_{11}\left(10^{-9} N s / V C\right)$ & 0 & 0 & 1.0 \\
\hline$\mu_{11}\left(10^{-6} N s^{2} / C^{2}\right)$ & 5.0 & -590.0 & 0 \\
\hline
\end{tabular}


The total volume fraction of piezoelectric phase and piezomagnetic phase is 0.6 . The change of effective electromagneto-elastic moduli with volume fraction of piezomagnetic phase $\lambda$ for the square and hexagonal arrays are shown in Fig. (3). The dilute, self-consistent, MoriTanaka, differential and generalized self-consistent methods have been extensively used for predicting the effective properties of composites. Generally, the results predicted by the generalized self-consistent method (GSCM) are in good agreement with the experiment data. As a comparison with the presented method, the results predicted by GSCM are also depicted in Fig. (3).

(a) Square
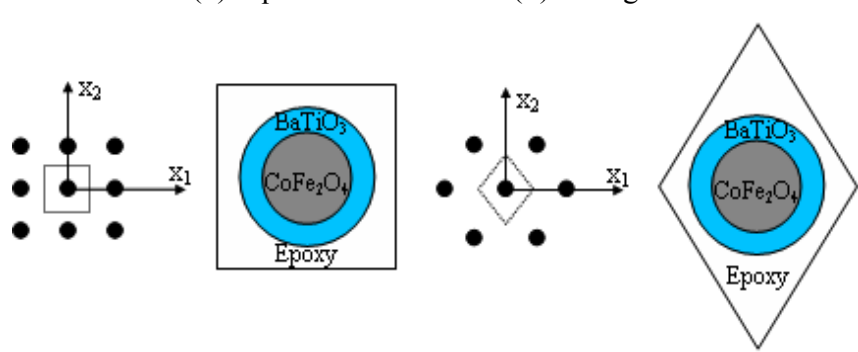

(b) Hexagonal

Fig. (2). Fiber square and hexagonal arrays and unit cell (threephase model).

(a) Effective elastic modulus

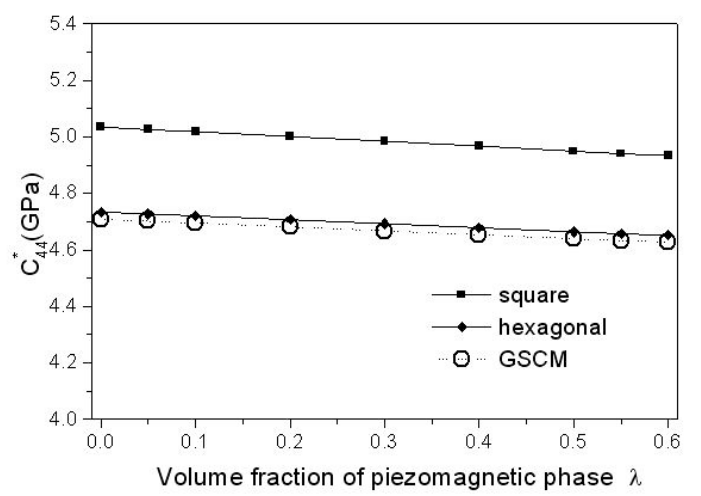

(b) Effective piezoelectric modulus

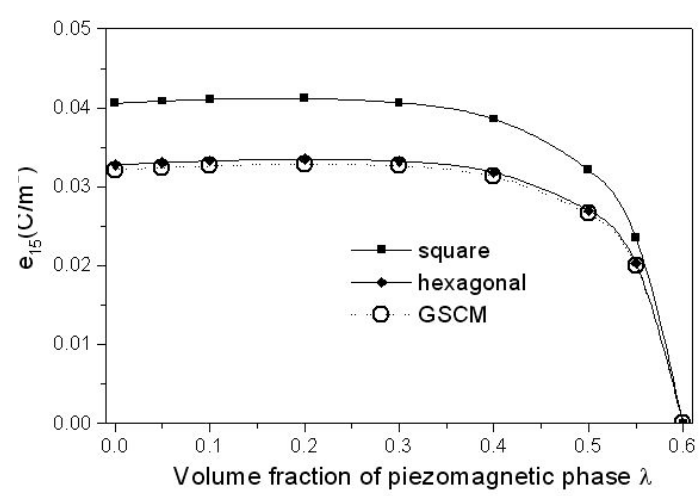

(Fig. 3) contd....

(c) Effective piezomagnetic modulus

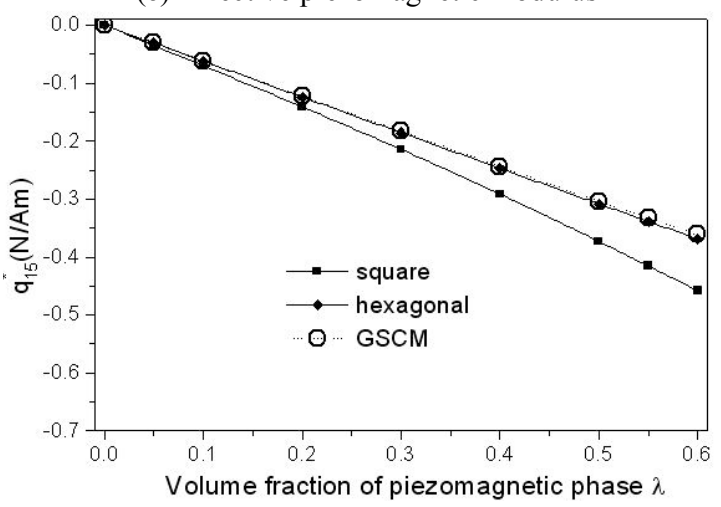

(d) Effective dielectric modulus

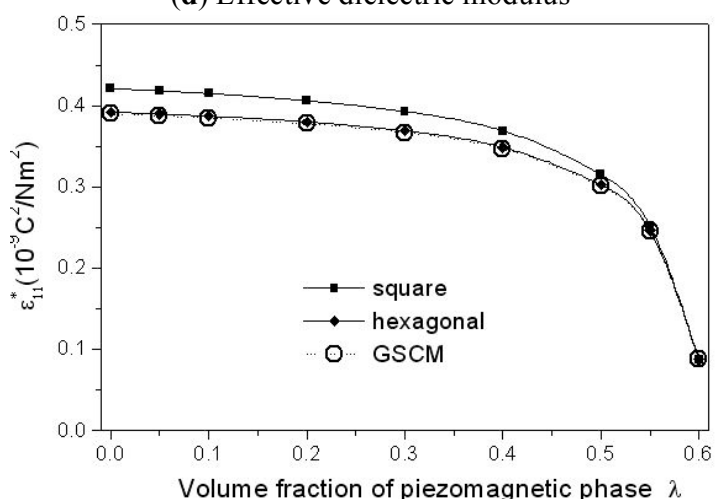

(e) Effective magneto-electric modulus

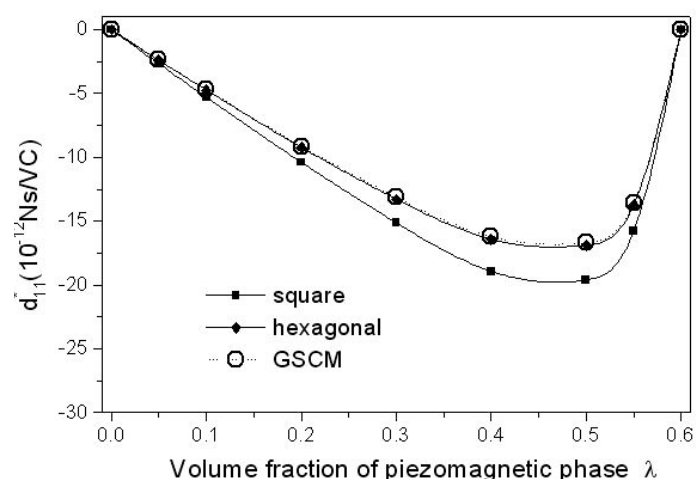

(f) Effective magnetic permeability modulus

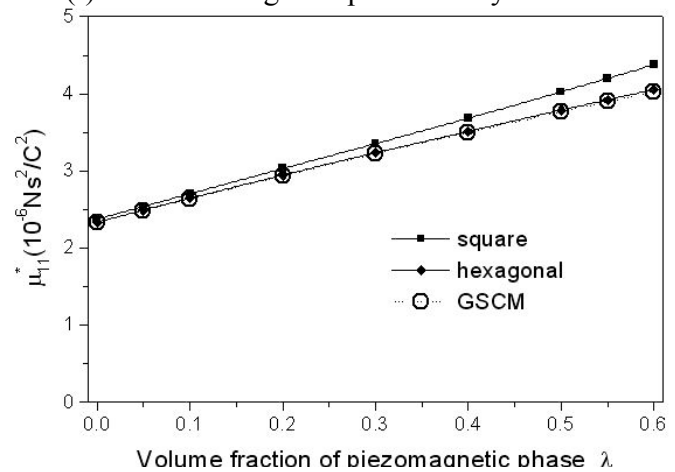

Fig. (3). Results and comparisons of effective electro-magnetoelastic moduli. 
From Fig. (3), it is seen that the results are different between square array and hexagonal array, some reasons for this behavior has been discussed by Pettermann and Suresh [7]. It is also seen that the results predicted by the GSCM are almost in exact agreement with those for the hexagonal array predicted by the present method. It appears that the GSCM reflects "the idealized even distribution" of inclusions and in the doubly periodic arrays the hexagonal array approaches most "the idealized even distribution". When $\lambda=0$ and $\lambda=0.6$, the reinforced phase is piezoelectric phase $\left(\mathrm{BaTiO}_{3}\right)$ or piezomagnetic phase $\left(\mathrm{CoFe}_{2} \mathrm{O}_{4}\right)$, there in no magneto-electric coupling effect, effective magneto-electric modulus is zero. When $0<\lambda<0.6$, the three-phase electro-magneto-elastic composite contain a non-zero magneto-electric modulus. When $\lambda$ is about 0.5 , the magneto-electric coupling effect is maximum.

Example 2: The hexagonal array of fibers is adopted in this example. Take the fiber and matrix as the piezoelectric phase $\mathrm{BaTiO}_{3}$ and the piezomagnetic phase $\mathrm{CoFe}_{2} \mathrm{O}_{4}$, respectively. A pure elastic coating Epoxy is between the fiber and matrix, as shown in Fig. (4). The dimensionless coating thickness $\delta=\left(R_{1}-R_{0}\right) / a=0.01$. The coating elastic modulus $\mathrm{C}_{44}^{\mathrm{C}}=\gamma \times 1.28 \mathrm{GPa}$ (different value of $\gamma$ denotes different coating elastic modulus). The variations of the electro-magneto-elastic effective moduli with $\gamma$ for different fiber volume fraction $\lambda$ are depicted in Fig. (5).

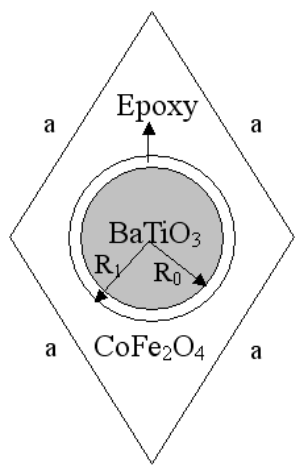

Fig. (4). An unit cell with Epoxy coating.

(a) Effective elastic modulus

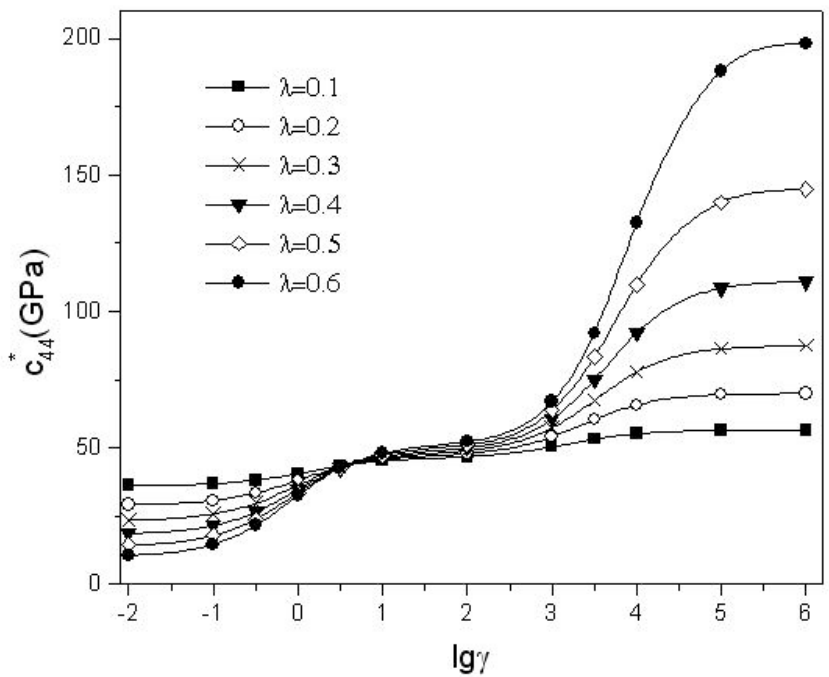

(b) Effective piezoelectric modulus

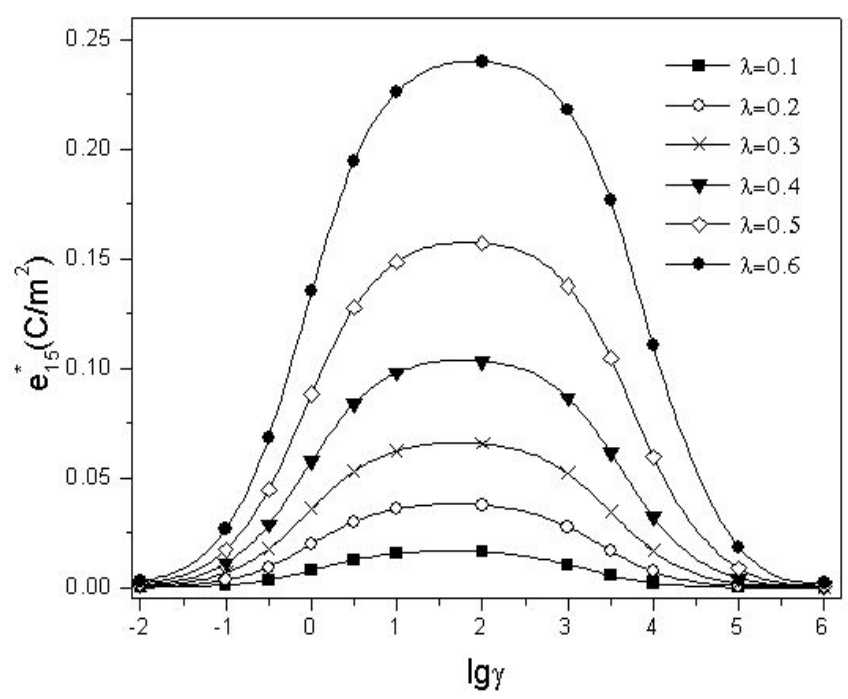

(c) Effective magneto-electric modulus

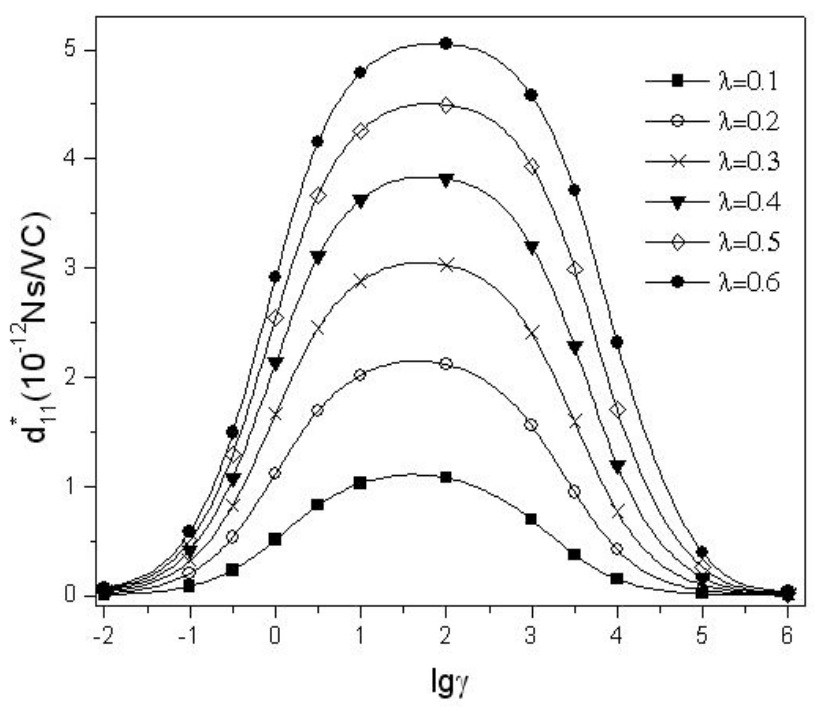

(d) Effective magnetic permeability modulus

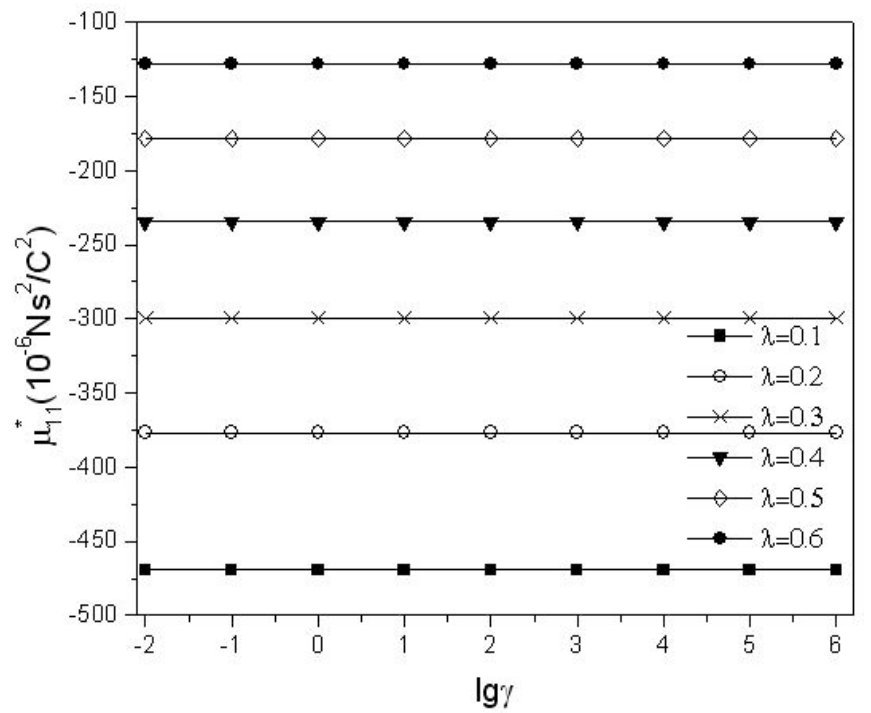


(Fig. 5) contd.....

(e) Effective piezomagnetic modulus

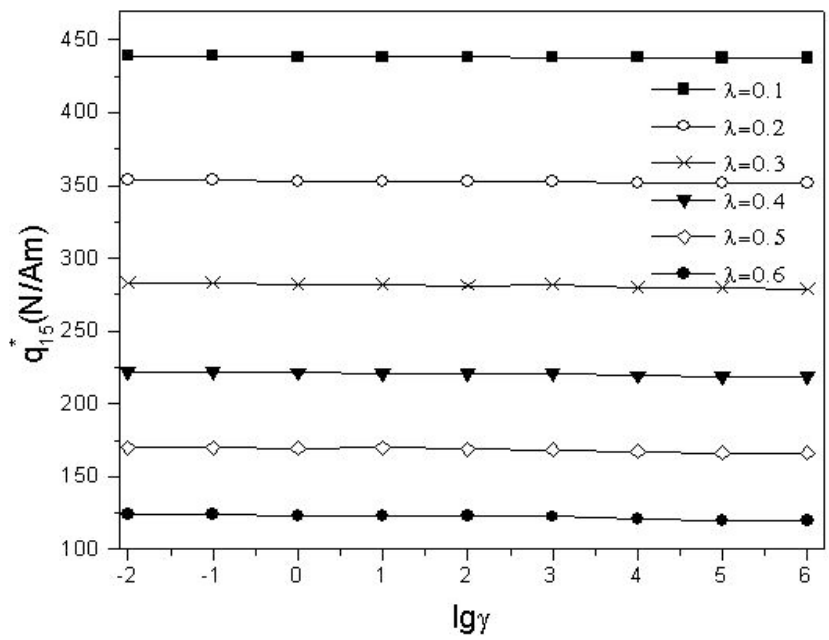

(f) Effective dielectric modulus

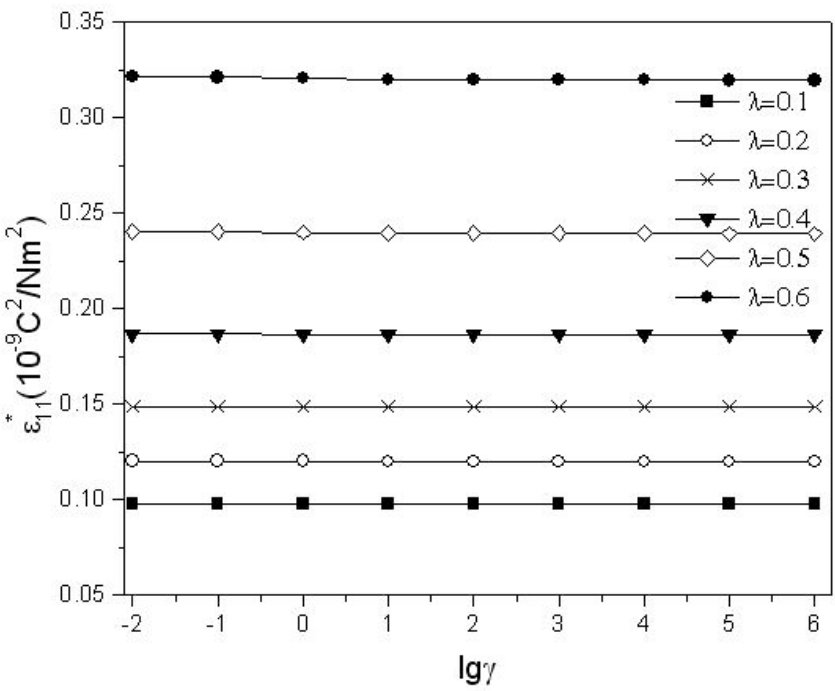

Fig. (5). Effect of the coating elastic moduli on the magnetoelectric effective moduli.

From Fig. (5), the effective elastic increase monotonously with the increase of the coating stiffness. The effective piezoelectric and magneto-electric moduli increase no monotonously with the increase of the coating stiffness, which can be interpreted by the fact that a stiff or a soft coating is disadvantageous to transfer strain from matrix (piezomagnetic phase $\mathrm{CoFe}_{2} \mathrm{O}_{4}$ ) to fibers (piezoelectric phase $\mathrm{BaTiO}_{3}$ ). When coating is too stiff or too soft, the strain in fibers is very little, and the effective piezoelectric and magneto-electric moduli are close zero. Numerical results also indicate that the coating stiffness has very little influence on the effective magnetic permeability, piezomagnetic and dielectric moduli.

\section{CONCLUSIONS}

For the electro-magneto-elastic composites with a doubly periodic parallelogram array of reinforced phases under farfield antiplane shear coupled with inplane electromagnetic load, a rigorous analytical method is developed by introducing the concepts of generalized eigenstrain integrated with the doubly quasi-periodic Riemann boundary problem. Numerical results show the difference between square array and hexagonal array of reinforced phases. A comparison of the present solution with results from GSCM demonstrates the efficiency and accuracy of the present method.

\section{ACKNOWLEDGMENTS}

The Committee of ICMSE 2011 acknowledges the support from Bentham for publishing the accepted paper in her OPEN Journals, the work is supported by the National Natural Science Foundation of China under Grant NNSFC 51078322 and the Natural Science Foundation of Hebei Province under Grant E2011203218.

\section{REFERENCES}

[1] Nan CW. Magnetoelastic effect in composites of piezoelectric and piezoelectric phases. Phys Rev B 1994; 50: 6082-88.

[2] Aboudi J. Micromechanical anslysis of fully coupled electromagneto-thermo-elastic multiphase composites. Smart Mater Struct 2001; 10: 867-77.

[3] Boyd JG, Lagoudas DC, Seo CS. Arrays of micro-electrodes and electromagnets for processing of electro-magneto-elastic multifunctional composite materials. SPIE 10th Annual International Symposium on Smart Structures, San Diego, USA, 2003.

[4] Lee J, Boyd JGIV, Lagoudas DC. Effective properties of threephase electro-magneto-elastic composites. Int J Eng Sci 2005; 43 : 790-825.

[5] Fang QH, Liu YW, Jiang CP. On the interaction between a generalized screw dislocation and circular-arc interfacial rigid lines in magnetoelectroelastic solids. Int J Eng Sci 2005; 43: 1011-31.

[6] Lu JK. Boundary Value Problems for Analytic Function, Singapare: World Scientific, 1993.

[7] Pettermann HE, Suresh S. A comprehensive unit cell model: study of coupled effects in piezoelectric 1-3 composites. Int J Solids Struct 2000; 37: 5447-64. 